\title{
Sum regression decomposition of spectral and angle-resolved Mueller-matrices from biological reflectors
}

\author{
Roger Magnusson, Hans Arwin, Enric Garcia-Caure, Kenneth Järrendahl and Razvigor \\ Ossikovski \\ Journal Article
}

\section{Tweet}

N.B.: When citing this work, cite the original article.

Original Publication:

Roger Magnusson, Hans Arwin, Enric Garcia-Caure, Kenneth Järrendahl and Razvigor Ossikovski, Sum regression decomposition of spectral and angle-resolved Mueller-matrices from biological reflectors, Applied Optics, 2016. 55(15), pp.4060-4065.

http://dx.doi.org/10.1364/AO.55.004060

Copyright: Optical Society of America

http://www.osa.org/

Postprint available at: Linköping University Electronic Press

http://urn.kb.se/resolve?urn=urn:nbn:se:liu:diva-111946

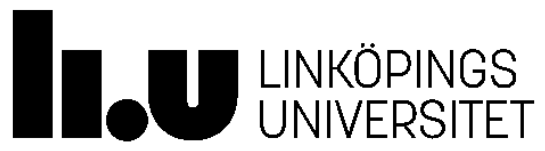




\title{
Sum regression decomposition of spectral and angle-resolved Mueller matrices from biological reflectors
}

\author{
Roger Magnusson ${ }^{1, *}$, Hans Arwin ${ }^{1}$, Enric Garcia-Caurel ${ }^{2}$, Kenneth Järrendahl ${ }^{1}$, And \\ RAZVIGOR OSSIKOVSKI ${ }^{2}$ \\ ${ }^{1}$ Department of Physics, Chemistry and Biology, Linköping University, SE-58183 Linköping, Sweden \\ ${ }^{2}$ LPICM, CNRS, Ecole Polytechnique, Université Paris - Saclay, FR-91128 Palaiseau, France \\ *Corresponding author: roger.magnusson@liu.se \\ Compiled May 2, 2016
}

We show spectroscopic Mueller-matrix data measured at multiple incidence angles of the scarab beetle C. aurata. The Mueller matrix is decomposed by method of regression decomposition and it is shown that this Mueller matrix can be decomposed into a set of two matrices representing one polarizer and one dielectric reflector. We also report on a tentative decomposition of the beetle $C$. argenteola using the same method. () 2016 Optical Society of America

OCIS codes: (310.5448) Polarization, other optical properties; (240.2130) Ellipsometry and polarimetry; (160.1435) Biomaterials.

http://dx.doi.org/10.1364/ao.XX.XXXXXX

\section{INTRODUCTION}

A Mueller matrix is a complete description of the linear optical response of a sample when light is reflected specularly off it or transmitted through it [1]. With Mueller-matrix spectroscopic ellipsometry (MMSE), the spectral and angle of incidence dependence of a Mueller matrix for a sample can be measured accurately [2] and can be used to extract optical and structural properties of samples composed of complex nanostructured materials. Mueller matrices are based on a Stokes vector description of light and thus have the benefit of being able to include not only interaction with polarized light but also with unpolarized and partially polarized light. MMSE is frequently used in analysis of bulk and thin film structures and during the last decade it has also been employed to study natural photonic structures [3, 4]. These results may be helpful in biomimicry as nature has long been a source of inspiration for developing and refining technologies in materials science. Structural coloring may serve as one example and mimicking of structures of morpho butterflies could lead to environmentally friendly long-lasting colors [5]. In this report we present studies on beetles in the Scarabaeidae family. The selected beetles show brilliant colors and in addition interesting polarization features. Mueller matrices measured on such beetles are of large interest to explore for biomimetics and for the understanding of the biological relevance of the observed polarization phenomena. Several species in the Scarabaeidae family have been studied by Hodgkinson [6], Goldstein [3] and our group $[4,7]$ to mention a few. Ellipticity, degree of polar- ization and other derived parameters have been reported [4] and Arwin et al. [7] also did optical modeling to determine structural parameters of the scutellum part of the exoskeleton of Cetonia aurata.

Mueller matrices are very rich in information about the sample properties and can also be analyzed by addressing depolarization. Cloude [8] showed that a depolarizing Mueller matrix can be represented by a sum of up to four non-depolarizing Mueller matrices weighted by the eigenvalues of the covariance matrix of the Mueller matrix. These eigenvalues are all positive for a physically realizable Mueller matrix and this, so called sum decomposition can be used to filter matrices and obtain a measure of experimental fidelity [9]. The result of the decomposition can also be used to describe a Mueller matrix as a set of basic optical elements having direct physical meaning, such as polarizers and retarders. Pioneering work on decomposition of Mueller-matrix images, including studies of beetles, was performed by Ossikovski et al. [10]. We have also previously [11] demonstrated this with Cloude as well as regression decomposition of Mueller-matrix spectra and images measured at near-normal incidence on C. aurata. Using Cloude decomposition we found that the experimentally determined Mueller matrix of C. aurata at near normal incidence decomposes into a set of a mirror and a circular polarizer. Those results were then the basis for a more stable regression decomposition where the result was confirmed.

Our objective in this work is to further develop the parameterization of Mueller matrices of beetles in terms of basic optical 
elements using regression decomposition. We generalize the previous decomposition by including angle-of-incidence depending Mueller matrices which leads to more complicated decompositions.

\section{EXPERIMENTAL DETAILS}

We have measured Mueller matrices of two species of scarab beetles: Cetonia aurata (Linnaeus, 1758) collected on the island Öland in Sweden and Chrysina argenteola (Bates, 1888) which is on loan from the Natural Museum of History in Stockholm, Sweden, but originates from Colombia. MMSE measurements were performed on the scutellum, which is a small triangular and relatively flat area on the dorsal side of the beetle. The incidence angles were in the range $15^{\circ}$ to $55^{\circ}$. The ellipsometer used is a dual rotating compensator ellipsometer $\left(\mathrm{RC} 2{ }^{\circledR}\right)$ from J.A. Woollam Co., Inc. equipped with focusing optics to reduce the spot size to $<100 \mu \mathrm{m}$. The spectral range of the instrument is 245$1690 \mathrm{~nm}$ but only data in the range $245-1000 \mathrm{~nm}$ are presented here. All calculations regarding decomposition was made using the lsqcurvefit function in Matlab.

\section{THEORY}

When the polarization state of light is described with a Stokes vector, the incoming and outgoing waves are related by a $4 \times 4$ Mueller matrix $\mathbf{M}$ as [1]

$$
\mathbf{S}_{\mathbf{o}}=\mathbf{M S}_{\mathbf{i}}
$$

where $\mathbf{S}_{\mathbf{i}}$ and $\mathbf{S}_{\mathbf{o}}$ are Stokes vectors for the incoming and the outgoing beams of light, respectively. If we expand the equation we get

$$
\left[\begin{array}{c}
I_{o} \\
Q_{o} \\
U_{o} \\
V_{o}
\end{array}\right]=\left[\begin{array}{cccc}
1 & m_{12} & m_{13} & m_{14} \\
m_{21} & m_{22} & m_{23} & m_{24} \\
m_{31} & m_{32} & m_{33} & m_{34} \\
m_{41} & m_{42} & m_{43} & m_{44}
\end{array}\right]\left[\begin{array}{c}
1 \\
Q_{i} \\
U_{i} \\
V_{i}
\end{array}\right]
$$

where $I, Q, U$ and $V$ denote the Stokes parameters [1] and $m_{i j}$ $(i, j=1,2,3,4)$ are the elements of $\mathbf{M}$. $\mathbf{M}$ and $\boldsymbol{S}_{\mathbf{i}}$ are normalized to $m_{11}$ and $I_{i}$, respectively. Based on this formalism we briefly describe sum decomposition and present some basic Mueller matrices used in this work.

\section{A. Regression sum decomposition}

Cloude has shown that a depolarizing Mueller matrix can be represented as a sum of up to four non-depolarizing matrices [8]. As an alternative to Cloude's method, regression decomposition can be used as recently shown for spectral data and Muellermatrix images on C. aurata [11]. An experimentally determined normalized Mueller matrix $\mathbf{M}$ is then decomposed into a sum of Mueller matrices $\mathbf{M}_{i}^{\text {reg }}$ representing optical devices such as polarizers, retarders and rotators.

$$
\mathbf{M}^{r e g}=a \mathbf{M}_{1}^{r e g}+b \mathbf{M}_{2}^{r e g}+c \mathbf{M}_{3}^{r e g}+d \mathbf{M}_{4}^{r e g}
$$

The coefficients $a, b, c$ and $d$ are fit parameters and the matrices $\mathbf{M}_{1}^{r e g}, \mathbf{M}_{2}^{r e g}, \mathbf{M}_{3}^{r e g}, \mathbf{M}_{4}^{\text {reg }}$ are defined beforehand. The decomposition can be made using matrices with fixed values on all elements or with generalized matrices whereby some matrix parameters can also be fitted. Later we will describe examples of such matrices that have been used in this paper. Any Muellermatrix decomposition based on Cloude's method will result in up to four trace-orthogonal matrices. The matrices possible to use in our regression-based decomposition are not necessarily trace-orthogonal and thus an infinite number of sum decompositions are possible. In fact, Eq. 3 is a special form of the arbitrary decomposition of a Mueller matrix [12] where four terms are included but they can be fewer or more depending on the complexity of $\mathbf{M}$. However, the number of fit parameters including those within the matrices can not exceed the degrees of freedom of $\mathbf{M}$. The number of non-zero matrix components in Eq. 2 can be determined from Cloude's decomposition. In the regression procedure, the Frobenius norm [13]

$$
F=\left\|\mathbf{M}-\mathbf{M}^{r e g}\right\|_{F}
$$

is minimized and the coefficients $a, b, c, d$ are all positive and $a+b+c+d=1$.

\section{B. Mueller matrices for rotated polarizing components}

If an optical device is rotated an angle $\alpha$ counterclockwise with respect to the $x$-axis in a reference Cartesian coordinate system when looking into the source, the Mueller matrix $\mathbf{M}^{\prime}$ of the rotated device will be

$$
\mathbf{M}^{\prime}=\mathbf{R}(-\alpha) \mathbf{M R}(\alpha)
$$

where $\mathbf{M}$ is the Mueller matrix of the unrotated device and $\mathbf{R}(\alpha)$ is given by [14]

$$
\mathbf{R}(\alpha)=\left[\begin{array}{cccc}
1 & 0 & 0 & 0 \\
0 & \cos 2 \alpha & \sin 2 \alpha & 0 \\
0 & -\sin 2 \alpha & \cos 2 \alpha & 0 \\
0 & 0 & 0 & 1
\end{array}\right]
$$

\section{Polarizers}

A polarizer will attenuate two orthogonal components of the electric field in a plane wave by different amounts. An ideal linear polarizer with its transmission axis in the $x$-direction is given by [1]

$$
\mathbf{M}_{P}^{\text {linear }}=\frac{1}{2}\left[\begin{array}{cccc}
1 & 1 & 0 & 0 \\
1 & 1 & 0 & 0 \\
0 & 0 & 0 & 0 \\
0 & 0 & 0 & 0
\end{array}\right]
$$

If such an ideal polarizer is rotated so that the transmission axis becomes oriented at an angle $\alpha$ from the $x$-axis, its Mueller matrix as obtained from Eq. 5 will be

$$
\mathbf{M}_{P}^{\text {linear }}(\alpha)=\frac{1}{2}\left[\begin{array}{cccc}
1 & \cos 2 \alpha & \sin 2 \alpha & 0 \\
\cos 2 \alpha & \cos ^{2} 2 \alpha & \sin 2 \alpha \cos 2 \alpha & 0 \\
\sin 2 \alpha & \sin 2 \alpha \cos 2 \alpha & \sin ^{2} 2 \alpha & 0 \\
0 & 0 & 0 & 0
\end{array}\right]
$$

A circular polarizer will attenuate left-handed and right-handed polarized light by different amounts and its Mueller matrix is 
given by

$$
\mathbf{M}_{P}^{\text {circular }}=\frac{1}{2}\left[\begin{array}{cccc}
1 & 0 & 0 & \pm 1 \\
0 & 0 & 0 & 0 \\
0 & 0 & 0 & 0 \\
\pm 1 & 0 & 0 & 1
\end{array}\right]
$$

where $m_{14}=m_{41}=1$ is for right-handed and -1 for left-handed polarization capability. Linear and circular polarizers are special cases of the more general elliptic polarizer and a general elliptical polarizer with azimuth angle equal to $0^{\circ}$ or $90^{\circ}$ has a Mueller matrix given by

$$
\mathbf{M}_{P}^{\text {elliptic }}(\varepsilon)=\frac{1}{2}\left[\begin{array}{cccc}
1 & \cos 2 \varepsilon & 0 & \sin 2 \varepsilon \\
\cos 2 \varepsilon & \cos ^{2} 2 \varepsilon & 0 & \cos 2 \varepsilon \sin 2 \varepsilon \\
0 & 0 & 0 & 0 \\
\sin 2 \varepsilon & \cos 2 \varepsilon \sin 2 \varepsilon & 0 & \sin ^{2} 2 \varepsilon
\end{array}\right]
$$

where $\varepsilon$ is the ellipticity angle of the major eigenpolarization [15]. For incident unpolarized light, the Stokes vector of emerging light moves along the meridian of the Poincaré sphere, passing through horizontal linear, right-handed circular, vertical linear and left-handed circular polarization states as $2 \varepsilon$ goes through values $0^{\circ}, 90^{\circ}, 180^{\circ}$ and $270^{\circ}$, respectively. With an arbitrary azimuth angle $\alpha$, Eqs. 5 and 10 lead to

$$
\mathbf{M}_{P}^{\text {elliptic }}(\varepsilon, \alpha)=\frac{1}{2}\left[\begin{array}{cccc}
1 & a b & a c & d \\
a b & a^{2} b^{2} & a^{2} b c & a b d \\
a c & a^{2} b c & a^{2} c^{2} & a c d \\
d & a b d & a c d & d^{2}
\end{array}\right]
$$

where $a=\cos 2 \varepsilon, b=\cos 2 \alpha, c=\sin 2 \alpha$ and $d=\sin 2 \varepsilon$. Eq. 11 corresponds to transmission mode but in reflection off a symmetric, reciprocal material it has been shown $[16,17]$ that the off-diagonal elements of the Jones matrix are anti symmetrical and therefore the Mueller matrix will satisfy the following symmetries: $m_{31}=-m_{13}, m_{32}=-m_{23}$ and $m_{34}=-m_{43}$ (see e.g. Garcia-Caurel et al. [18]) and we obtain

$$
\mathbf{M}_{P, R}^{\text {elliptic }}(\varepsilon, \alpha)=\frac{1}{2}\left[\begin{array}{cccc}
1 & a b & a c & d \\
a b & a^{2} b^{2} & a^{2} b c & a b d \\
-a c & -a^{2} b c & a^{2} c^{2} & -a c d \\
d & a b d & a c d & d^{2}
\end{array}\right]
$$

\section{Isotropic reflector}

An isotropic reflector used at oblique incidence introduces retardation $\delta$ and diattenuation $\cos 2 \psi$ between the electric field components in two orthogonal directions parallel and perpendicular to the plane of incidence [2].

$$
\mathbf{M}_{R}^{I s o}=\left[\begin{array}{cccc}
1 & -\cos 2 \psi & 0 & 0 \\
-\cos 2 \psi & 1 & 0 & 0 \\
0 & 0 & \sin 2 \psi \cos \delta & \sin 2 \psi \sin \delta \\
0 & 0 & -\sin 2 \psi \sin \delta & \sin 2 \psi \cos \delta
\end{array}\right]
$$

Two special cases are noted. One occurs when $\delta$ is $180^{\circ}$ and $\psi$ is $45^{\circ}$. Eq. 13 then becomes

$$
\mathbf{M}_{M}=\left[\begin{array}{cccc}
1 & 0 & 0 & 0 \\
0 & 1 & 0 & 0 \\
0 & 0 & -1 & 0 \\
0 & 0 & 0 & -1
\end{array}\right]
$$

which is an ideal mirror. The second case occurs when $\delta$ is $180^{\circ}$ and $\psi$ is $0^{\circ}$ and then Eq. 13 becomes

$$
\mathbf{M}_{L P}=\left[\begin{array}{cccc}
1 & -1 & 0 & 0 \\
-1 & 1 & 0 & 0 \\
0 & 0 & 0 & 0 \\
0 & 0 & 0 & 0
\end{array}\right]
$$

which is a linear polarizer with its absorption axis parallel to the plane of incidence.

\section{RESULTS AND DISCUSSION}

\section{A. Regression sum decomposition of Mueller matrices of $\boldsymbol{C}$. aurata}

Mueller matrices measured on C. aurata at incidence angles in the range $15^{\circ}$ to $55^{\circ}$ with a step size of $1^{\circ}$ in the spectral range $245 \mathrm{~nm}$ to $1000 \mathrm{~nm}$ are summarized in a contour plot in Fig. 1. Below $500 \mathrm{~nm}$ and above $600 \mathrm{~nm}$ all off-diagonal block elements

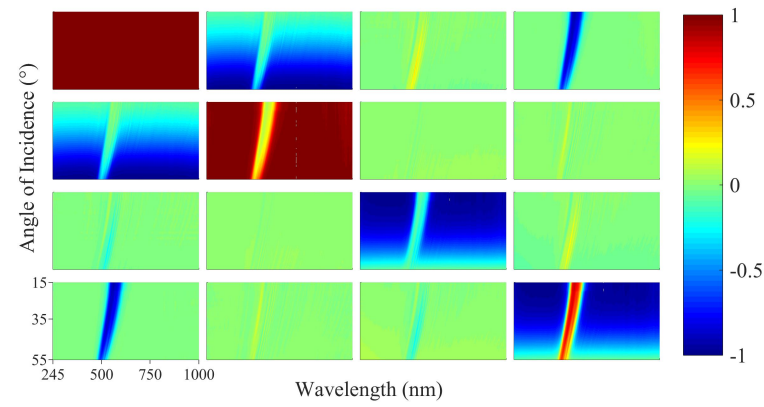

Fig. 1. A contour plot of experimentally determined $4 \times 4$ Mueller matrices of $C$. aurata.

as well as $m_{34}$ and $m_{43}$ are almost constant and close to zero and the rest of the elements have monotonously changing values with increasing incidence angle. Between $500 \mathrm{~nm}$ and $600 \mathrm{~nm}$, however, many elements show dramatic changes. In this region the Mueller matrix is depolarizing and can therefore be sum decomposed according to Cloude [8] as shown in our previous report [11]. We call this region the circularly polarizing regime and will be referred to as the $P_{C}$-regime. Within the $P_{C}$-regime the cuticle will reflect incident unpolarized light as near-circular polarized at small angles of incidence $\theta$ with an ellipticity decreasing with $\theta$. In our previous work based on near-normal incidence data, we used an ansatz with a decomposition into a circular polarizer and a dielectric mirror. A circular polarizer is a special case of an elliptical polarizer and a dielectric mirror can be represented by a retarder with $\delta=180^{\circ}$ with the conventions used. Here we expand the circular polarizer to an elliptical polarizer and the mirror to a isotropic reflector according to Eqs. 10 and 13 , respectively. 
We apply a regression analysis according to Eqs. 3 and 4 with the ansatz

$$
\mathbf{M}^{\text {reg }}=a(\lambda, \theta) \mathbf{M}_{P, R}^{\text {elliptic }}(\alpha, \varepsilon)+b(\lambda, \theta) \mathbf{M}_{R}^{\text {Iso }}(\psi, \delta)
$$

where $\lambda$ and $\theta$ are wavelength and incidence angle, respectively; $\mathbf{M}_{P, R}^{\text {elliptic }}$ is the Mueller matrix of a general normalized elliptical polarizer with variable ellipticity angle $\varepsilon$ and azimuth angle $\alpha$ as in Eq. 12 and $\mathbf{M}_{R}^{I s o}$ is the Mueller matrix of an isotropic reflector as in Eq. 13 with variable diattenuation $\psi$ and retardation $\delta$. The coefficients $a$ and $b$ are limited to vary between 0 and 1 with the constraint $a+b=1$. The result of the regression can be seen in Fig. 2. The coefficients $a$ and $b$ in Figs 2(a) and 2(d) both show quite featureless variations except in the $P_{C}$-regime. Coefficient $a$ shows values close to zero outside the $P_{C}$-regime whereas inside the $P_{C}$-regime values reach 0.9 . Since the sum of $a$ and $b$ is unity, coefficient $b$ is close to one outside the $P_{C}$-regime and 0.1 inside. This behavior in combination with the choice of matrices $\mathbf{M}_{P, R}^{\text {elliptic }}$ and $\mathbf{M}_{R}^{\text {Iso }}$ in Eq. 16 is consistent with previous results [11].

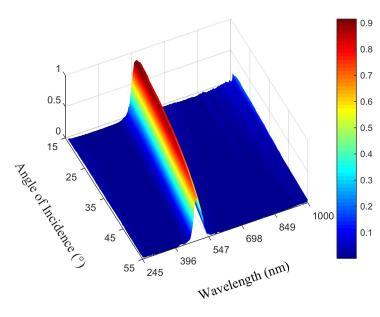

(a) Coefficient $a$

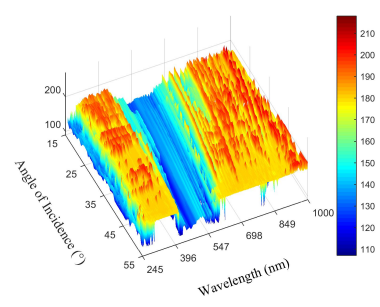

(b) $\varepsilon$

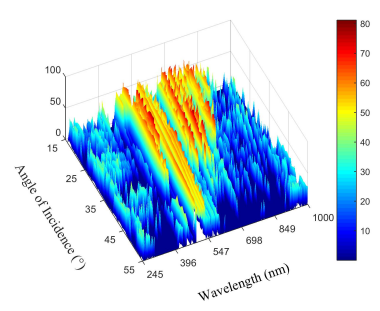

(c) $\alpha$

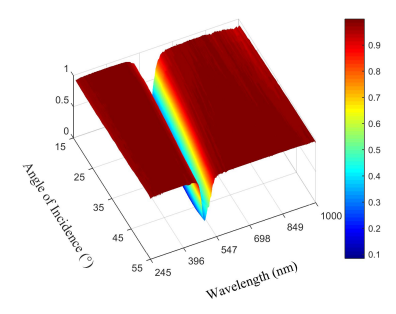

(d) Coefficient $b$

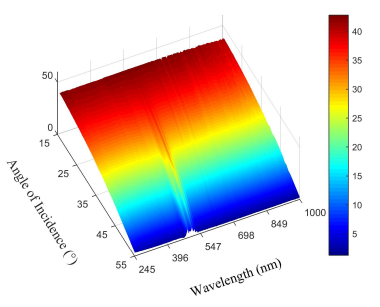

(e) $\psi$

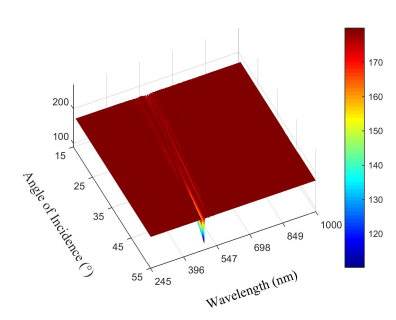

(f) $\delta$
Fig. 2. Result of regression analysis of data from C. aurata using the ansatz in Eq. 16.

\section{A.1. Outside the $P_{C}$-regime}

Outside the $P_{C}$-regime the contribution from the elliptical polarizer to the optical properties is approximately zero and the values of $\varepsilon$ and $\alpha$ in Figs. 2(b) and 2(c), respectively, are therefore of little relevance.

The values of $\psi$ and $\delta$ of the isotropic reflector can be seen in Figs. 2(e) and 2(f), respectively. The value of $\psi$ is a function of wavelength and varies from $43^{\circ}$ at near normal incidence to $0^{\circ}$ at larger incidence angles and $\delta$ is constant at $180^{\circ}$.

When the values at near-normal incidence are put into Eq. 13 the isotropic reflector becomes a mirror as in Eq. 14, and for large incidence angles the same isotropic reflector is a linear polarizer described in Eq. 15.

\section{A.2. Inside the $P_{C}$-regime}

The optical properties inside the $P_{C}$-regime can be described by a sum of the elliptical polarizer and the retarder. Here the values of $\psi$ and $\delta$ of the retarder show some small variations as seen in Figs. 2(e) and 2(f). Compared to the value outside $P_{C}$-regime, there is a small increase in $\psi$ of approximately $10^{\circ}$ for incidence angles above $35^{\circ}$ in a very narrow band in the center of the $P_{C}$-regime. $\delta$ varies from $180^{\circ}$ down to $160^{\circ}$ for incidence angles below approximately $40^{\circ}$ and it drops to $110^{\circ}$ in the same narrow band in the center of the $P_{C}$-regime close to $55^{\circ}$.

The angle $\varepsilon$ and the azimuthal rotation $\alpha$ of the polarizer both have a strong dependence on wavelength and oscillate rapidly if viewed at a fixed angle of incidence. $\varepsilon$ ( Fig. 2(b)) varies from approximately $130^{\circ}$ to $150^{\circ}$ with a tendency to lower values at smaller incidence angles. $\alpha$ (Fig. $2(\mathrm{c}))$ shows small variations between $30^{\circ}$ and $60^{\circ}$ throughout the $P_{C}$-regime. When these values are put into Eq. 12 and multiplied by its weight factor $a$, the matrix (Fig. 3) will be similar to that of a left-handed circular polarizer. However, all elements will have non-zero values inside the $P_{C}$-regime, particularly in rows 1 and 4 and in columns 1 and 4 . This deviation from a pure circular polarizer is likely due to in-plane anisotropy in the sample under inspection.

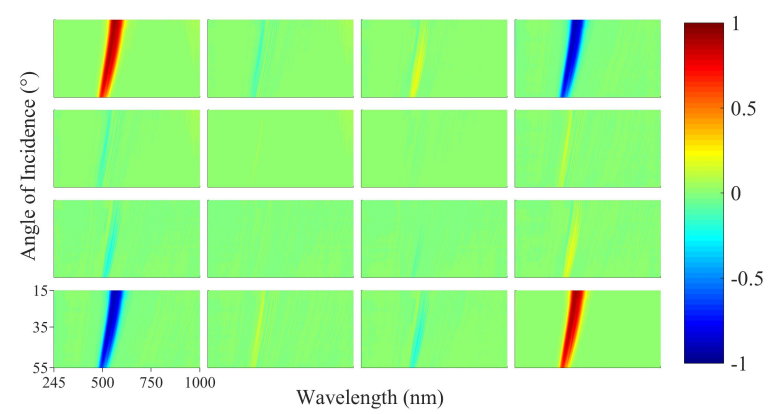

Fig. 3. The first term of Eq. $16, a(\lambda, \theta) \mathbf{M}_{P, R}^{\text {elliptic }}(\alpha, \varepsilon)$.

The values from Figs. 2(e) and 2(f) are put into Eq. 13 and multiplied by its weight factor $b$ and the resulting matrix can be seen in Fig. 4 . Here we see very small values inside the $P_{C^{-}}$ regime and outside it has the characteristics of a reflection from a dielectric material, i.e. Eq. 13 with $\delta=180^{\circ}$.

When the matrices in Figs. 3 and 4 are added according to Eq. 16 the result is very close to the experimental matrix. The differences between the experimental data and $\mathbf{M}^{\text {reg }}$ after decomposition can be seen in Fig. 5. Given the complexity of the biological reflector the agreement is generally very good and the differences between the experimental data and the result of the regression is within \pm 0.08 for all $\theta$ and $\lambda$. From Figs. 2(a) and $2(\mathrm{~d})$ the conclusion can be drawn that outside the $P_{C}$-regime the polarizing properties for all $\lambda$ and $\theta$ of $C$. aurata are completely described by an isotropic reflector with $\psi$ according to Fig. 2(e) and $\delta=180^{\circ}$. Inside the $P_{C}$-regime the situation is slightly more complex and the polarizing properties cannot be completely described by a single, easily identifiable Mueller matrix. Here the properties are described by a sum of an elliptical 


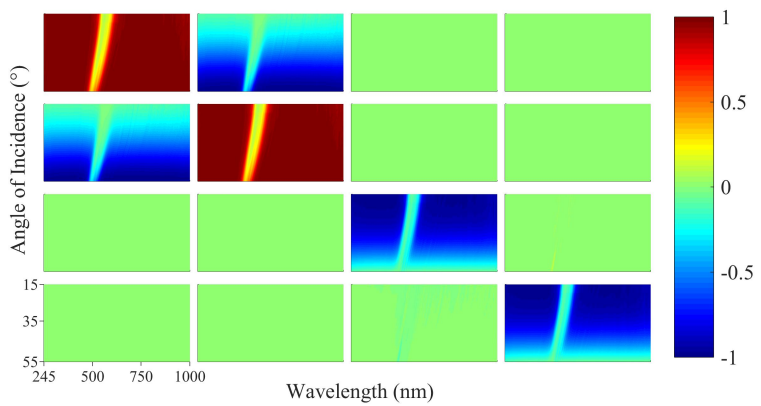

Fig. 4. The second term of Eq. $16, b(\lambda, \theta) \mathbf{M}_{R}^{I s o}(\psi, \delta)$.

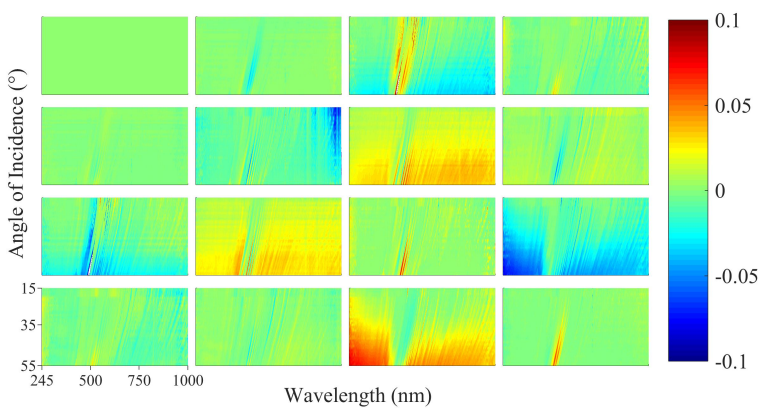

Fig. 5. The difference between the experimental Mueller matrix and $\mathbf{M}^{\text {reg }}$ as calculated by Eq. 16 for C. aurata.

polarizer and an isotropic reflector where the contribution from the reflector is only in the diagonal of the Mueller matrix and by a small amount. The main contribution comes from the polarizer where the values are such that it is close to a circular polarizer. However, the elements $m_{12}, m_{13}, m_{21}, m_{24}, m_{31}, m_{34}, m_{42}$ and $m_{43}$ are non-zero which indicates that some anisotropy in the surface-plane is involved. The sensitivity to such anisotropy depends on the spot size which will increase along the plane of incidence with larger incidence angles. This has been discussed in previous work [11] and the effect is seen in Fig. 5 as an increasing value in the matrix elements with increasing incidence angle.

\section{B. Regression sum decomposition of Mueller matrices of C. argenteola}

Mueller matrices of $C$. argenteola were recorded at incidence angles in the range $15^{\circ}$ to $55^{\circ}$ with a step size of $1^{\circ}$ in the spectral range $245 \mathrm{~nm}$ to $1000 \mathrm{~nm}$ and are summarized in a contour plot in Fig. 6. The broadband reflector C. argenteola exhibits left-handed polarization features for small $\theta$ seen as the large blue regions in Mueller matrix elements $m_{14}$ and $m_{41}$, and righthanded polarization features for large $\theta$, seen as the small red regions close to $55^{\circ}$ in the same elements. A notable feature in Fig. 6 is also the interference oscillations which are seen in most elements as rapid variations in wavelength dependence. We here present only rudimentary trials on regression decomposition of $C$. argenteola using the same ansatz as was used for $C$. aurata (i.e. Eq. 16). The resulting parameters are presented in Fig. 7. However, the fit is not satisfactory as the Frobenius norm using Eq. 4 has values up to 0.3 in some regions for several of the elements, and up to 0.5 in element $m_{44}$. We also find that a Cloude decomposition results in three (or more depending on the incidence angle; see our previous work [11]) non-zero eigen-

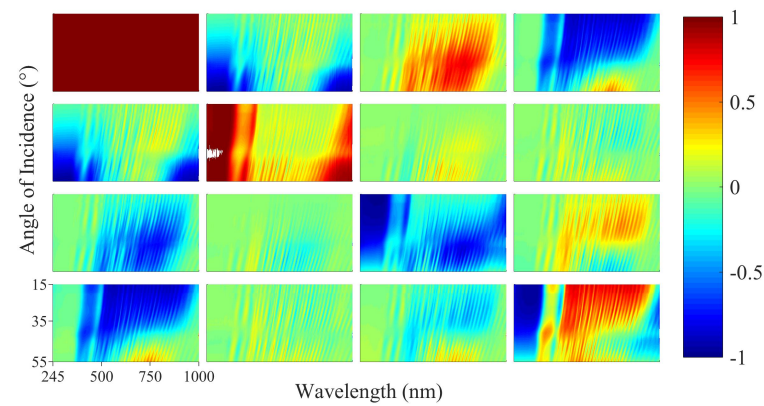

Fig. 6. A contour plot of the experimentally determined Mueller matrix of $C$. argenteola.

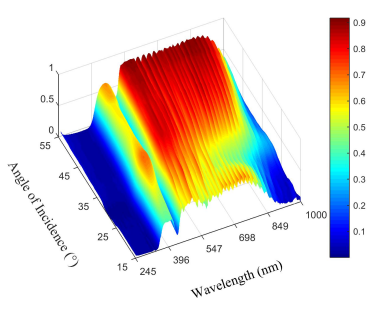

(a) Coefficient $a$

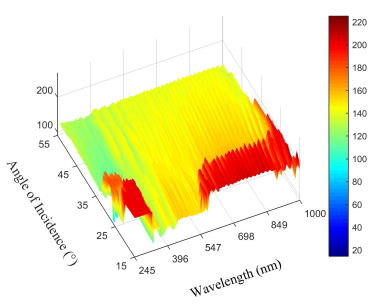

(b) $\varepsilon$

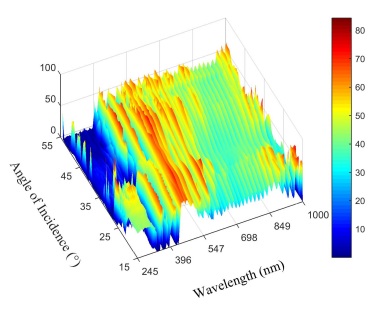

(c) $\alpha$

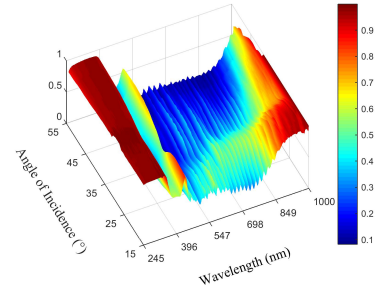

(d) Coefficient $b$

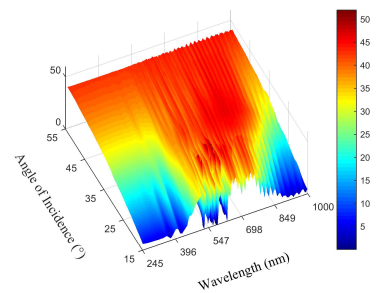

(e) $\psi$

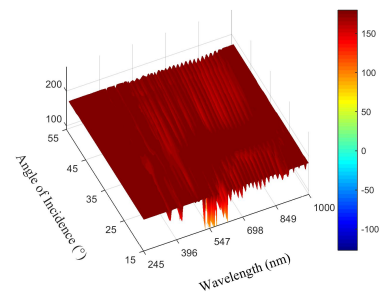

(f) $\delta$
Fig. 7. Result of regression analysis of data from C. argenteola using the ansatz in Eq. 16.

values for C. argenteola compared to only two for C. aurata as seen in Fig. 8. This means that more than two matrices should be included in a regression analysis and not only two as was used to derive the parameters in Fig. 7. One reason for the larger complexity in the analysis of $C$. argenteola data is that the pronounced interference patterns seen both in primary data in Fig. 6 and in eigenvalues in Fig. 8 shows that its cuticle is more transparent compared to that of $C$. aurata. The probe depth is therefore larger and structures deeper inside the cuticle are probed. In a broadband reflector like $C$. argenteola the Mueller matrix then becomes more complex as structures have different pitches at different depths. Similar effects has been observed and analyzed in the beetle Cotinis mutabilis [19]. Work is in progress to determine 


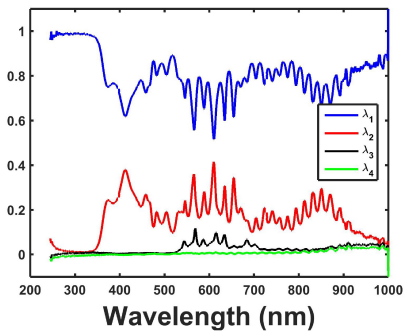

(a)

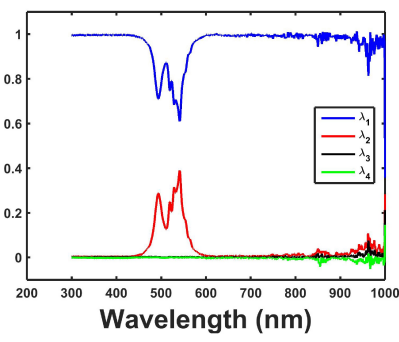

(b)
Fig. 8. The eigenvalues from a Cloude decomposition of (a) C. argenteola and (b) C. aurata, both at $45^{\circ}$ incidence angle.

additional matrices suitable for the regression decomposition of C. argenteola data.

\section{Final remarks}

We have presented a regression-based decomposition analysis of Mueller matrices measured as a function of wavelength and incidence angle. A sum of an elliptical polarizer and an isotropic reflector has been fitted to these experimental Mueller matrices. In the case of $C$. aurata this works well. In the case of $C$. argenteola, however, the ansatz presented in Eq. 16 does not work which is explained by the fact that a Cloude decomposition reveals that additional matrices are needed in the ansatz and further investigations will be performed.

\section{FUNDING INFORMATION}

Swedish Research Council; Carl Tryggers Foundation; Knut and Alice Wallenberg foundation is acknowledged for support to instrumentation.; H.A. acknowledges financial support from the Swedish Government Strategic Research Area in Materials Science on Functional Materials at Linköping University (Faculty Grant SFO Mat LiU No 2009 00971);; The authors thank Jan Landin for providing specimens of $C$. aurata and the Swedish Museum of Natural History for lending specimen of $C$. argenteola.

\section{REFERENCES}

1. E. Collett, Polarized Light: Fundamentals and Applications (Marcel Dekker, 1993).

2. H. Fujiwara, Spectroscopic Ellipsometry: Principles and Applications (John Wiley \& Sons, 2007).

3. D. H. Goldstein, "Polarization properties of scarabaeidae," Appl. Opt. 45, 7944-7950 (2006).

4. H. Arwin, R. Magnusson, J. Landin, and K. Järrendahl, "Chirality-induced polarization effects in the cuticle of scarab beetles: 100 years after Michelson," Philos. Mag. 92, 1583-1599 (2012).

5. A. Saito, "Material design and structural color inspired by biomimetic approach," Sci. Technol. Adv. Mater. 12, 064709 (2011).

6. I. Hodgkinson, S. Lowrey, L. Bourke, A. Parker, and M. W. McCall, "Mueller-matrix characterization of beetle cuticle: polarized and unpolarized reflections from representative architectures," Appl. Opt. 49, 45584567 (2010).

7. H. Arwin, T. Berlind, B. Johs, and K. Järrendahl, "Cuticle structure of the scarab beetle Cetonia aurata analyzed by regression analysis of Muellermatrix ellipsometric data," Opt. Express 21, 22645-22656 (2013).

8. S. R. Cloude, "Conditions for the physical realisability of matrix operators in polarimetry," in "Polarization Considerations for Optical Systems II," , vol. 1166, R. A. Chipman, ed. (1990), vol. 1166, pp. 177-187.
9. R. Ossikovski, M. Anastasiadou, S. Ben Hatit, E. Garcia-Caurel, and A. De Martino, "Depolarizing Mueller matrices: how to decompose them?" phys. status solidi (a) 205, 720-727 (2008).

10. R. Ossikovski, M. Foldyna, C. Fallet, and A. De Martino, "Experimental evidence for naturally occurring nondiagonal depolarizers," Opt Lett 34, 2426-2428 (2009).

11. H. Arwin, R. Magnusson, E. Garcia-Caurel, C. Fallet, K. Järrendahl, M. Foldyna, A. De Martino, and R. Ossikovski, "Sum decomposition of Mueller-matrix images and spectra of beetle cuticles," Opt. Express 23, 1951-1966 (2015).

12. J. J. Gil, "Polarimetric characterization of light and media," The European Physical Journal - Applied Physics 40, 1-47 (2007).

13. G. H. Golub and C. F. V. Loan, Matrix Computations (JHU Press, 1996), 3rd ed.

14. R. M. A. Azzam and N. M. Bashara, Ellipsometry and Polarized Light (Elsevier, Amsterdam, 1999).

15. D. S. Kliger, J. W. Lewis, and C. E. Randall, Polarized Light in Optics and Spectroscopy (Academic Press, 1990), 2nd ed.

16. H. C. van de Hulst, Light Scattering by Small Particles (Dover Publications, New York, 1981), reprint ed.

17. L. Li, "Symmetries of cross-polarization diffraction coefficients of gratings," J. Opt. Soc. Am. A 17, 881-887 (2000).

18. E. Garcia-Caurel, R. Ossikovski, M. Foldyna, A. Pierangelo, B. Drevillon, and A. De Martino, "Ellipsometry at the Nanoscale," in "Ellipsometry at the Nanoscale," , M. Losurdo and K. Hingerl, eds. (Springer Berlin Heidelberg, Berlin, Heidelberg, 2013).

19. A. Mendoza-Galván, E. Muñoz-Pineda, K. Järrendahl, and H. Arwin, "Evidence for a dispersion relation of optical modes in the cuticle of the scarab beetle Cotinis mutabilis " Optical Materials Express 4, 2484 (2014). 